\title{
Charge density wave instabilities of type-II Weyl semimetals in a strong magnetic field
}

\author{
Maximilian Trescher, ${ }^{1}$ Emil J. Bergholtz, ${ }^{2}$ Masafumi Udagawa, ${ }^{3}$ and Johannes Knolle ${ }^{4}$ \\ ${ }^{1}$ Dahlem Center for Complex Quantum Systems and Institut für Theoretische Physik, Freie Universität Berlin, \\ Arnimallee 14, 14195 Berlin, Germany \\ ${ }^{2}$ Department of Physics, Stockholm University, AlbaNova University Center, 10691 Stockholm, Sweden \\ ${ }^{3}$ Department of Physics, Gakushuin University, Mejiro, Toshima-ku, Tokyo 171-8588, Japan \\ ${ }^{4}$ TCM Group, Cavendish Laboratory, J. J. Thomson Avenue, Cambridge CB3 OHE, United Kingdom
}

(Received 8 June 2017; published 1 November 2017)

\begin{abstract}
Shortly after the discovery of Weyl semimetals, properties related to the topology of their bulk band structure have been observed, e.g., signatures of the chiral anomaly and Fermi arc surface states. These essentially single particle phenomena are well understood, but whether interesting many-body effects due to interactions arise in Weyl systems remains much less explored. Here, we investigate the effect of interactions in a microscopic model of a type-II Weyl semimetal in a strong magnetic field. We identify a charge density wave (CDW) instability even for weak interactions stemming from the emergent nesting properties of the type-II Weyl Landau level dispersion. We map out the dependence of this CDW on magnetic field strength. Remarkably, as a function of decreasing temperature, a cascade of CDW transitions emerges and we predict characteristic signatures for experiments.
\end{abstract}

DOI: 10.1103/PhysRevB.96.201101

Introduction. The theory of Weyl fermions in condensed matter systems-semimetals where conduction and valence bands have nondegenerate touching points - has a long and intriguing history, bringing ideas originally developed in the context of particle physics into the realm of materials physics [1-5]. Recently, the experimental discovery of a Weyl semimetal was reported by various groups [6-8], soon followed by transport measurements demonstrating the chiral anomaly $[9,10]$ as well as the connection of Fermi arc surface states to chiral bulk modes in strong magnetic fields [11].

Much recent attention has focused on type-II Weyl semimetals [12] whose linear dispersion is so strongly tilted [13-16] that it forms electron and hole pockets. Several materials in this class, including $\mathrm{WTe}_{2}[17], \mathrm{WP}_{2}[18,19]$, and $\mathrm{Mo}_{x} \mathrm{~W}_{1-x} \mathrm{Te}_{2}$ [20], have in parallel attracted ample attention due to their remarkable magnetotransport properties. While a single particle analysis reveals a novel twist on the chiral anomaly in type-II Weyl semimetals [21-23], there is no commonly accepted explanation of the observed magnetotransport properties nor an understanding of whether they are at all rooted in the topological properties of these materials, highlighting the need for an understanding of many-body effects.

Exotic interaction effects in Weyl semimetals [13,24], some taking place only in systems with tilted Weyl cones [13], have recently been explored. More conventional phenomena such as density wave instabilities have so far only been discussed in type-I Weyl semimetals where, however, they require a significant critical interaction strength at zero magnetic field [25-28], consider the chemical potential away from the Weyl node [29], or only appear in a magnetic field as effective onedimensional instabilities of the chiral mode [30-32]. Further spin ordering in Weyl semimetals has been studied [33].

Here, we show that the electron- and holelike pockets of the overtilted cones in type-II Weyl semimetals generically render these systems much more susceptible to interaction effects. In particular, we show that in a magnetic field the Landau level dispersion acquires nestinglike features between a large number of Landau level bands which triggers a charge density wave (CDW) transition for small interactions. As this emergent weak coupling instability in a magnetic field neither requires perfect particle-hole compensation nor nesting of the zero-field band structure, we argue that CDW phases are a common property of the high-field regime of type-II Weyl semimetals.

Our starting point is a simple microscopic Hamiltonian $H=H_{0}+H_{\text {int }}$ with a quadratic lattice model $H_{0}$ featuring Weyl nodes and a local density-density interaction $H_{\text {int }}$. We show that in a field, a weak coupling intracone CDW instability with a wave vector related to the electron and hole pocket separation appears. Our qualitative discussion is corroborated by a microscopic calculation for which we derive a continuum description. Going beyond lowest order in the momenta is necessary to describe closed electron and hole pockets. We note that this is in general crucial for a correct low-energy description in type-II Weyl systems.

This Rapid Communication is structured as follows: First, we introduce a simple microscopic model of a type-II Weyl semimetal. Calculating the dispersion of Landau levels in a magnetic field both in the lattice and in the corresponding continuum description, we give an intuitive argument for a CDW instability based on emergent nesting features. Second, we develop the mean-field theory for generic interactions in a type-II Weyl cone in a magnetic field. Third, we present self-consistent CDW solutions as a function of temperature and magnetic field. Finally, we discuss implications for experiments.

The model. We first concentrate on the noninteracting band structure governed by

$$
\begin{aligned}
H_{0}(\mathbf{k})= & \left(M-\cos k_{x}-\cos k_{y}\right) \sigma^{x}+\sin k_{y} \sigma^{y} \\
& +\sin k_{z} \sigma^{z}+\left(t_{1} \sin k_{z}+t_{2} \sin 2 k_{z}\right) \sigma^{0},
\end{aligned}
$$

in which the tilt of the Weyl cones can be easily tuned to feature electron and hole pockets, e.g., at $M=1, t_{1}+2 t_{2}>1$, this model has type-II Weyl nodes at $\mathbf{k}_{W}=( \pm \pi / 2,0,0)$. Since we 
are interested in intracone instabilities, we can expand around one of the cones, yielding a low-energy continuum description

$$
\begin{aligned}
H_{0}^{\mathrm{eff}}(\mathbf{k})= & \pm k_{x} \sigma^{x}+k_{y} \sigma^{y}+\left(k_{z}-\frac{1}{6} k_{z}^{3}\right) \sigma^{z} \\
& +\left(\left(t_{1}+2 t_{2}\right) k_{z}-\frac{1}{6}\left(t_{1}+8 t_{2}\right) k_{z}^{3}\right) \sigma^{0} .
\end{aligned}
$$

The momenta $k_{i}(i=x, y, z)$ are in the range $-\pi<k_{i} \leqslant \pi$ and measured in $1 / a_{0}$, where $a_{0}$ is the lattice spacing. We have omitted an overall prefactor $\hbar v$ which sets the energy scale in terms of Fermi velocity $v$. Importantly, we have expanded up to $O\left(k_{x}^{2}, k_{y}^{2}, k_{z}^{5}\right)$ and included the next-to-leading-order term in $k_{z}$. We are not aware that this has been done before but it is crucial for a correct low-energy description with closed electron and hole pockets.

Throughout this Rapid Communication we assume the realistic but exemplary values of $\hbar v=1 \mathrm{eV} \AA$ and $a_{0}=7 \AA$. The term proportional to $t_{2}$ is included to get smoother electron and hole pockets [see Fig. 1, top panel] and to elucidate how longer-range hopping terms can be included in the low-order expansion. Our effective model inherits the perfect compensation between electron and hole pockets present in our tight-binding model and which is also a prominent feature of $\mathrm{WTe}_{2}$. However, as discussed below, such perfect compensation is not a crucial ingredient of our CDW mechanism.

It is worth mentioning that there are also type-I nodes at $\mathbf{k}=( \pm \pi / 2,0, \pi)$ in this lattice model. However, these are unimportant for our considerations and could easily be removed at the price of a having a more complex lattice model.

We concentrate on magnetic fields along the $\mathbf{z}$ tilt direction of the cones leading to flat Landau levels in the $k_{x}, k_{y}$ plane dispersing only along the $k_{z}$ direction. Then we can introduce the field via the usual vector potential A minimally coupled to the crystal momentum $\Pi=\hbar \mathbf{k}-\frac{e}{c} \mathbf{A}$ and work with the usual ladder operators in the Landau level (LL) basis [see Supplemental Material (SM) [34] for details], such that the spectrum is given by the eigenvalues of

$$
\hat{H}_{0}^{\mathrm{eff}}=\left(-\eta k_{z}+\gamma k_{z}^{3}\right) \sigma_{0}+\left(k_{z}+\beta k_{z}^{3}\right) \sigma_{z}+\frac{\sqrt{2 n}}{l_{B}} \sigma_{x},
$$

with the Landau level index $n>0$ and the magnetic length $l_{B}=\sqrt{\frac{\hbar}{e B}}$. The parameters $\eta=-\left(t_{1}+2 t_{2}\right), \beta=-\frac{1}{6}, \gamma=$ $-\frac{1}{6}\left(t_{1}+8 t_{2}\right)$ are directly related to our lattice model, with $t_{1}=-0.8, t_{2}=-0.6$ for concreteness throughout this work.

Furthermore, we obtain the transformation relating our original sublattice creation operators $c_{A / B, n, p, k_{z}}$, with $p$ labeling the degenerate states within each LL, to new operators $a / b_{n, p, k_{z}}$ for the electron- and holelike bands,

$$
\left(\begin{array}{l}
a_{n, p, k_{z}} \\
b_{n, p, k_{z}}
\end{array}\right)=\underbrace{\left(\begin{array}{cc}
u\left(k_{z}, n, B\right) & v\left(k_{z}, n, B\right) \\
-v\left(k_{z}, n, B\right) & u\left(k_{z}, n, B\right)
\end{array}\right)}_{\hat{U}\left(k_{z}, n, B\right)}\left(\begin{array}{l}
c_{A, n, p, k_{z}} \\
c_{B, n, p, k_{z}}
\end{array}\right),
$$

with $u\left(k_{z}, n, B\right)^{2}+v\left(k_{z}, n, B\right)^{2}=1$. Their dispersions are given by

$$
E_{a / b}\left(k_{z}, n\right)=-\eta k_{z}+\gamma k_{z}^{3} \pm \sqrt{\left(k_{z}+\beta k_{z}^{3}\right)^{2}+\frac{2}{l_{B}^{2}}|n|},
$$
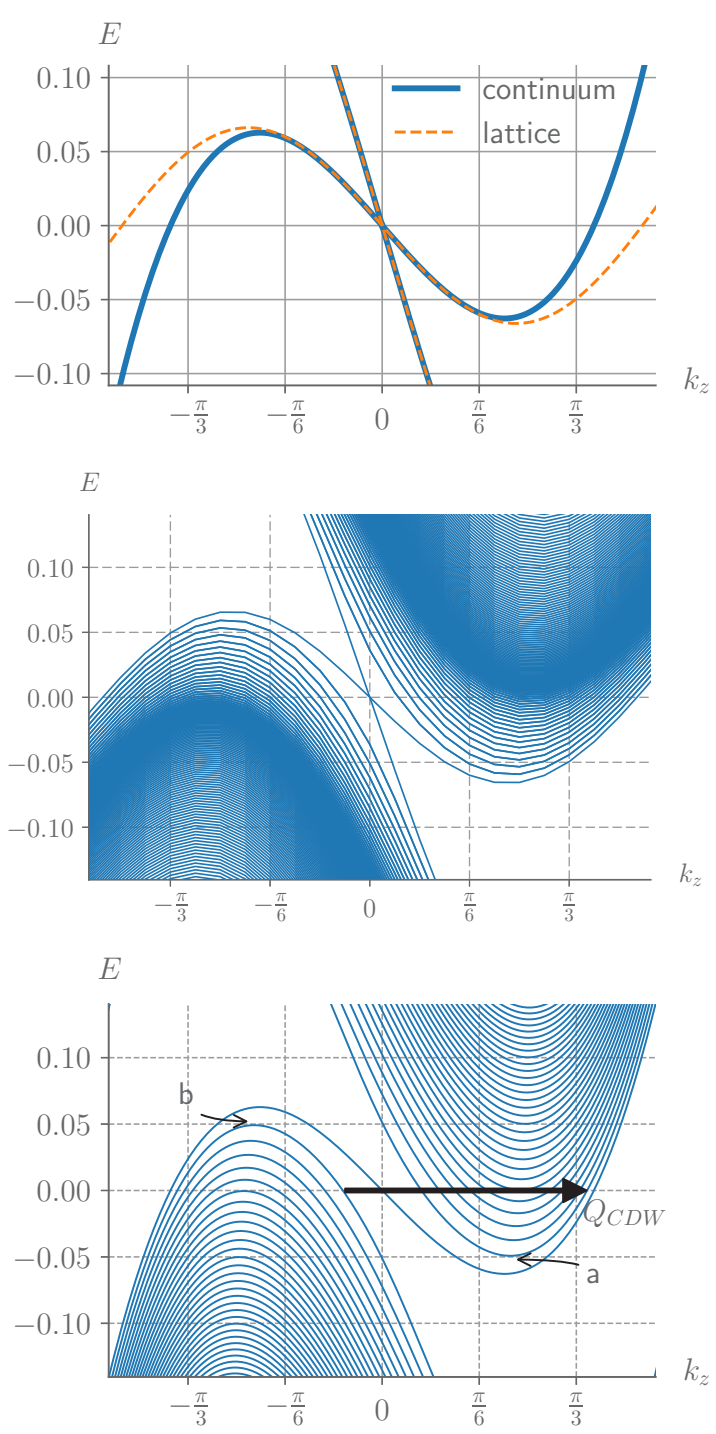

FIG. 1. Comparison of the dispersion of the lattice model and the continuum theory without magnetic field (top panel). The dispersion of the lattice model in a magnetic field is shown in the middle panel (with two Weyl cones at different $k_{x}$ superposed) and the corresponding dispersion of the continuum theory (single cone) in the bottom panel. The parameters of the lattice model are $t_{1}=-0.8$, $t_{2}=-0.6, a_{0}=7 \AA$, and the parameters of the low-energy theory are chosen to match the third-order expansion of the lattice model. The magnetic field strength for the bottom row is $B=86 \mathrm{~T}$.

for $n \neq 0$, and for $n=0$ the chiral level is given by

$$
E_{k_{z}, 0}=(1-\eta) k_{z}+(\beta+\gamma) k_{z}^{3} .
$$

The LL dispersions are shown in the bottom panel of Fig. 1 which can be directly compared to the corresponding numerical tight-binding calculation in a field displayed in the middle panel. Both make apparent one of the main findings of our work - the size and shape of the two inverted pockets almost exactly match when shifted by the arrow $Q_{\mathrm{CDW}}$ indicated in Fig. 1. These nesting features between entire electron and hole pockets, $E_{a}\left(k_{z}\right) \approx-E_{b}\left(k_{z}+Q_{\mathrm{CDW}}\right)$, are, for example, well established in parent compounds of 
iron-based superconductors, where they lead to density wave instabilities even for small interactions [35]. This is of course similar to the usual one-dimensional Peierls instability, which is cut off here by the broken inversion symmetry, but arguably more general.

A direct calculation of the corresponding LL- and pocketresolved susceptibility (see Fig. S1 in the SM) confirms the qualitative picture: (i) A dominant peak at $Q_{\mathrm{CDW}}$ appears in the interband component connecting electron and hole pockets; (ii) the peak is maximal for scattering between bands with the same LL index $n$; and (iii) due to a small asymmetry between the pockets, which also depends on the LL index, nesting is not perfect, which cuts off a true singularity of the susceptibility and introduces a peculiar field dependence. Note that in the case of imperfect particle-hole compensation, the pockets would be shifted in energy with respect to each other, leading to dominant nesting between branches with different LL indices, but the overall picture remains valid.

Mean-field ansatz. To study the formation of a CDW we add interactions to the single particle theory presented above. Due to the multiband nature, even the simplest contact interaction (strength $U$ ) is a sufficient approximation for a short-range interaction, as we are interested in the coupling between two different bands. We project the contact interaction to LLs,

$$
H_{\text {int }}=\frac{U}{2} \sum_{\substack{n_{1}, n_{2}, n_{3}, n_{4}, p_{1}, p_{2}, k_{z}, k_{z}^{\prime}, q_{x}, q_{y}, q_{z}}} e^{i q_{y}\left(p_{1}-p_{2}-q_{x}\right)} J_{n_{4}, n_{1}}(\mathbf{q}) J_{n_{3}, n_{2}}(-\mathbf{q}) \sum_{\alpha, \beta=A, B} c_{\alpha, n_{1}, p_{1}, k_{z}}^{\dagger} c_{\beta, n_{2}, p_{2}, k_{z}^{\prime}}^{\dagger} c_{\beta, n_{3}, p_{2}+q_{x}, k_{z}^{\prime}+q_{z}} c_{\alpha, n_{4}, p_{1}-q_{x}, k_{z}-q_{z}},
$$

which introduces additional momentum dependence [36] (see SM).

From the main peaks of bare susceptibility we know that the leading CDW instability arises between electron and hole bands with the very same LL index $n$. This allows us to simplify the problem considerably by only considering interactions with all $n_{1,2,3,4}=n$ equal. Hence the different Landau levels decouple, and we perform the following computations for a fixed Landau level index and combine results for different Landau levels later. Furthermore, since the nesting connects the different branches (with creation operators $a$ and $b)$, we are interested in a CDW in $\left\langle a^{\dagger} b\right\rangle$. We introduce the generic CDW wave vector $\mathbf{Q}=\left(Q_{x}, Q_{y}, Q_{z}\right)$ to formulate the general mean-field theory using the ansatz $\left\langle a_{p, k_{z}}^{\dagger} b_{p-q_{x}, k_{z}-q_{z}}\right\rangle=\Delta\left(k_{z}, \mathbf{Q}\right) e^{-i p Q_{y}} e^{i Q_{y} q_{x} / 2} \delta\left(q_{x}-Q_{x}\right) \delta\left(q_{z}-Q_{z}\right)$.

Our focus is on CDWs along the $k_{z}$ direction and therefore we concentrate on CDW vectors $\mathbf{Q}=(0,0, Q)$. We decouple the interaction in the usual way, which allows us to write the Hamiltonian in the bilinear form for each LL,

$$
H_{\mathrm{MF}, n}\left(p, k_{z}\right)=\left(\begin{array}{ll}
a_{p, k_{z}}^{\dagger} & b_{p, k_{z}-Q}^{\dagger}
\end{array}\right)\left(\begin{array}{cc}
E_{a}\left(p, k_{z}\right) & P\left(k_{z}\right) \\
P\left(k_{z}\right)^{*} & E_{b}\left(p, k_{z}-Q\right)
\end{array}\right)\left(\begin{array}{c}
a_{p, k_{z}} \\
b_{p, k_{z}-Q}
\end{array}\right) .
$$

The details of the derivation of the off-diagonal elements $P$ in terms of the projected interaction matrix elements [37] and the order parameter $\Delta$ are given in the SM. There, we also show that our ansatz gives real electron densities [38]. Knowing $P$, we obtain self-consistent solutions for $\Delta$ numerically and thereby determine whether or not the system supports a CDW.

As a check, we have confirmed that the wave vector corresponding to the smallest critical interaction for a meanfield CDW transition indeed coincides with the main peak of the bare susceptibility at $Q=Q_{\mathrm{CDW}}$.

Results: Cascade of CDW transitions in temperature. While an independent determination of the CDW wave vector ( $Q_{\mathrm{CDW}}$ ) for each Landau level is possible, we choose a common $Q_{\mathrm{CDW}}$ for all Landau levels to account for the inevitable inter-Landau-level coupling, which we neglected in our approximation. This global $Q_{\mathrm{CDW}}$ is obtained by maximizing the number of gapped levels, and hence represents the energetically most favorable configuration.

In Fig. 2 we show the numerical results for the critical temperature of the CDW transition at different magnetic fields and per Landau level $n$. It becomes clear that in lowering the temperature more and more, the Landau levels undergo the phase transition, hence we observe a cascade of successive $C D W$ transitions. It is important to keep in mind that the magnetic field also changes the spacing between Landau levels and thereby the number of Landau levels crossing the Fermi energy (the degeneracy in each level increases accordingly).
To account for this, we count the total number of gapped levels at each temperature and magnetic field strength and compute the fraction of this number compared to the number of Landau levels crossing the Fermi energy in the corresponding noninteracting system. This fraction is shown in the bottom panel of Fig. 2 and turns out to be roughly independent of field.

Experimental signatures. Our scenario implies thermodynamic signatures of a high-field phase transition, e.g., in specific heat or magnetization (see the inset in Fig. 3). The CDW real-space modulation of the electronic density $\rho(\mathbf{r}) \propto$ $\cos \left(Q_{\mathrm{CDW}} \cdot r_{z}\right)$ should be detectable via X-ray scattering.

In addition, the suppression of electronic states around the chemical potential entails clear experimental signatures and should be observable, for example, via scanning tunneling microscopy (STM) measurements. In Fig. 3 we compare the energy-resolved density of states (DOS) as a function of magnetic field between a noninteraction system (top panel) and the system with weak interactions (bottom panel). We observe a striking difference at zero energy (near the Weyl point) where the CDW clearly leads to a strong suppression of the DOS. As not all Landau levels are gapped by the CDW, for small interactions there is some residual DOS in this region, and hence it is not a complete gap.

Note that the DOS oscillates due to the discreteness of Landau levels. We expect that the corresponding quantum oscillations in thermodynamic observables survive even in regimes in which the CDW opens a full gap [39]. 


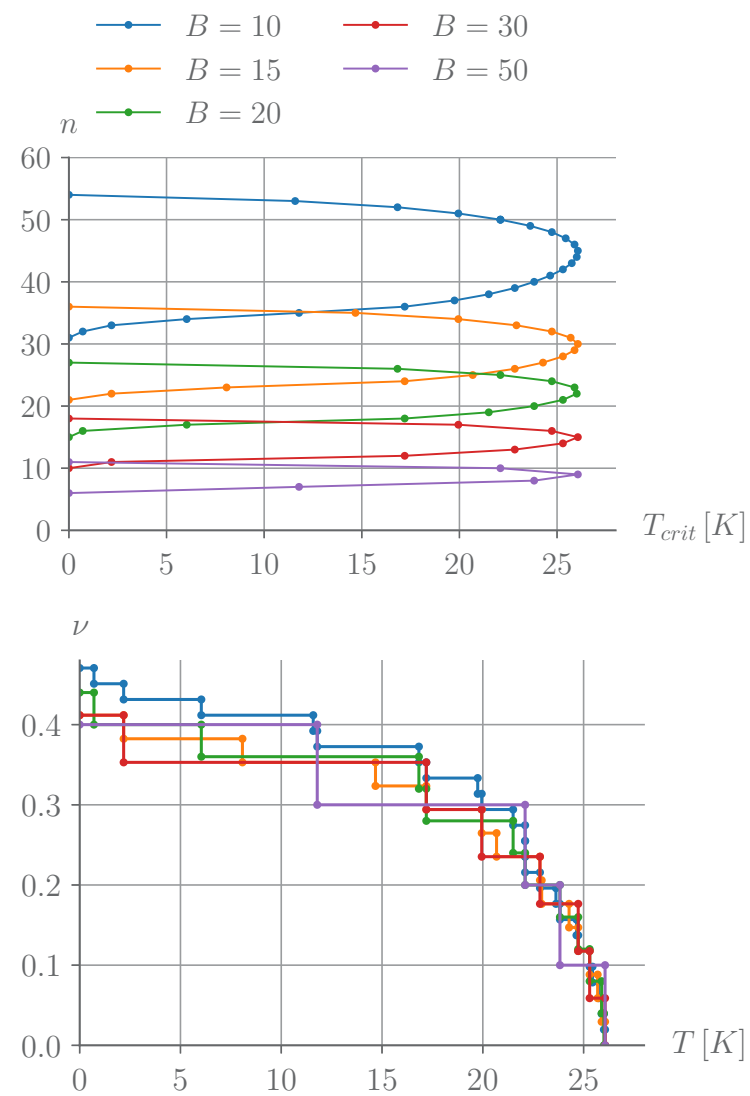

FIG. 2. Top panel: Highest critical temperature per Landau level for fixed interaction strength $U=0.025$ and fixed wave vector $Q_{\mathrm{CDW}}=1.48$ (see discussion in the main text). Bottom panel: Fraction of levels crossing the Fermi energy (when no interaction is considered) that are gapped by a CDW. When lowering the temperature, there is a cascade of consecutive CDW transitions, leading to an increased fraction of gapped levels. Parameters are the same as for the top panel.

Finally, if the magnetic field is not aligned in the direction of the tilt, as chosen in our setup, the electron- and holelike pockets of the LLs disappear [21], leading to a characteristic suppression of the CDW.

Discussion. We introduced an exemplary lattice model of a type-II Weyl semimetal and obtained a low-energy description that takes finite electron and hole pockets into account. From this we identified emergent nesting properties that occur between electron- and holelike Landau level branches once the Weyl semimetal is placed in a magnetic field. We developed a general mean-field theory of a Weyl semimetal in a field, confirming the intuitive picture of a nesting induced CDW instability. The self-consistent calculations allowed us to trace its dependence on temperature and magnetic field.

Here, we focused on the most relevant parts, i.e., intraLandau-level couplings between different branches. While we made plausible why these approximations should be valid, a more quantitative analysis of the inter-Landau-level couplings poses interesting questions for future research, as well as a full lattice calculation. We have pointed out several clear experimental signatures of the field-induced CDW transition, e.g., in thermodynamics, STM, and x rays.
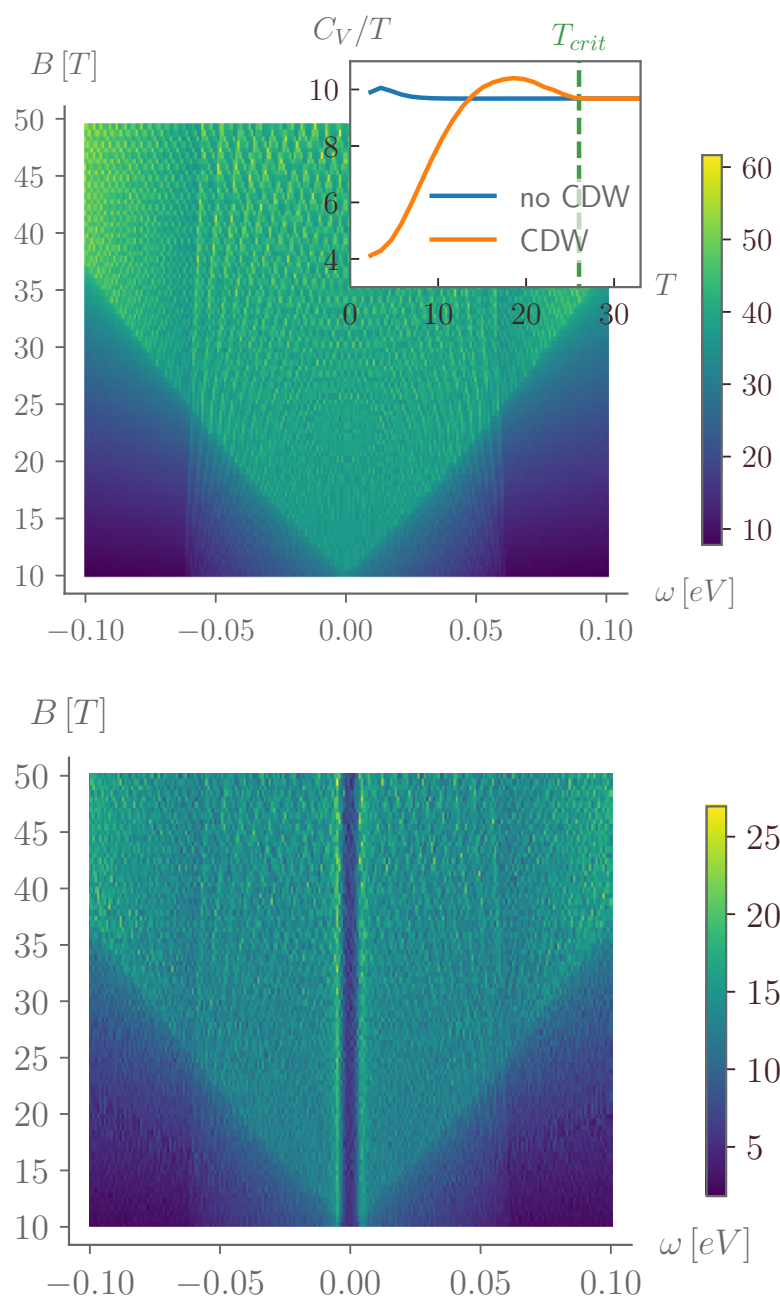

FIG. 3. DOS as a function of of magnetic field strength and energy, summed over the 50 lowest Landau levels. When including interactions (bottom panel, numerical result obtained at $T=4.6 \mathrm{~K}$ ), a gap opens, compared to the case without interactions (top panel). Inset: Temperature dependence of heat capacity $C_{V} / T$ (arbitrary units) at fixed magnetic field of $B=20 \mathrm{~T}$. As expected, $C_{V} / T$ is constant (blue line) without the $\mathrm{CDW}$, but by taking the $\mathrm{CDW}$ into account (orange line) deviations are clearly visible below the critical temperature indicated by the dashed green line.

The reduction of the DOS would also lead to an increased magnetoresistance, which is a feature of great interest in many type-II Weyl systems. A similar mechanism has been proposed to explain the magnetoresistance properties of graphite [40-42]. Considering the parallel alignment of the tilt and the magnetic field, our scenario is only directly applicable to $\mathrm{WP}_{2}[18,19]$, while the geometry is different in the case of $\mathrm{WTe}_{2}$ [17] and $\mathrm{Mo}_{x} \mathrm{~W}_{1-x} \mathrm{Te}_{2}$ [20]. A more detailed study of transport properties is desirable and left for future research. However, if our scenario is mutatis mutandis applicable to the nonsaturating magnetoresistance of these materials as well, there are some immediate consequences: Most saliently, it would be in contrast to the semiclassical picture suggested in Ref. [17] which relies on strict particle-hole symmetry. It is supported by the fact that the magnetotransport of 
$\mathrm{WTe}_{2}$ has an unusual temperature dependence and that $\mathrm{MoTe}_{2}$ with similar properties is far from particle-hole compensated [43]. Note that the number of LLs crossing the Fermi level is determined by the magnetic field component $B^{\perp}$ projected along the tilt direction. Hence, a magnetoresistance from LL formation of the form $\left(B^{\perp}\right)^{2}$ suggests a $\cos ^{2} \theta$ angular dependence similar to measurements on $\mathrm{WP}_{2}$ [19].

Finally, we note that for larger interactions the LL spectrum is fully gapped, which should lead to a concomitant three-dimensional Hall plateau [44] similar to other systems with density-wave-induced three-dimensional Hall effects $[45,46]$.

Acknowledgments. This work was supported by Emmy Noether program (BE 5233/1-1) of the Deutsche Forschungsgemeinschaft, the Swedish Research Council (VR), and the Wallenberg Academy Fellows program of the Knut and Alice Wallenberg Foundation. J.K. is supported by the Marie Curie Programme under EC Grant Agreement No. 703697.
[1] H. Weyl, Elektron und Gravitation. I, Z. Phys. 56, 330 (1929).

[2] G. E. Volovik, The Universe in a Helium Droplet (Oxford University Press, Oxford, U.K., 2009).

[3] S. Murakami, Phase transition between the quantum spin Hall and insulator phases in 3D: Emergence of a topological gapless phase, New J. Phys. 9, 356 (2007).

[4] X. Wan, A. M. Turner, A. Vishwanath, and S. Y. Savrasov, Topological semimetal and Fermi-arc surface states in the electronic structure of pyrochlore iridates, Phys. Rev. B 83, 205101 (2011).

[5] A. A. Burkov and L. Balents, Weyl Semimetal in a Topological Insulator Multilayer, Phys. Rev. Lett. 107, 127205 (2011).

[6] S. Xu, I. Belopolski, N. Alidoust, M. Neupane, G. Bian, C. Zhang, R. Sankar, G. Chang, Z. Yuan, C. Lee, S. Huang, H. Zheng, J. Ma, D. S. Sanchez, B. Wang, A. Bansil, F. Chou, P. P. Shibayev, H. Lin, S. Jia, and M. Z. Hasan, Discovery of a Weyl fermion semimetal and topological Fermi arcs, Science 349, 613 (2015).

[7] B. Q. Lv, H. M. Weng, B. B. Fu, X. P. Wang, H. Miao, J. Ma, P. Richard, X. C. Huang, L. X. Zhao, G. F. Chen, Z. Fang, X. Dai, T. Qian, and H. Ding, Experimental Discovery of Weyl Semimetal TaAs, Phys. Rev. X 5, 031013 (2015).

[8] L. Lu, Z. Wang, D. Ye, L. Ran, L. Fu, J. D. Joannopoulos, and M. Soljačić, Experimental observation of Weyl points, Science 349, 622 (2015).

[9] X. Huang, L. Zhao, Y. Long, P. Wang, D. Chen, Z. Yang, H. Liang, M. Xue, H. Weng, Z. Fang et al., Observation of the Chiral-Anomaly-Induced Negative Magnetoresistance in 3D Weyl Semimetal TaAs, Phys. Rev. X 5, 031023 (2015).

[10] C. Zhang, S. Xu, I. Belopolski, Z. Yuan, Z. Lin, B. Tong, G. Bian, N. Alidoust, C. Lee, S. Huang et al., Signatures of the Adler-Bell-Jackiw chiral anomaly in a Weyl fermion semimetal, Nat. Commun. 7, 10735 (2016).

[11] J. G. Ashvin and J. Analytis, Transport evidence for Fermiarc-mediated chirality transfer in the Dirac semimetal $\mathrm{Cd}_{3} \mathrm{As}_{2}$, Nature (London) 535, 266 (2016).

[12] A. A. Soluyanov, D. Gresch, Z. Wang, Q. Wu, M. Troyer, X. Dai, and B. A. Bernevig, Type-II Weyl semimetals, Nature (London) 527, 495 (2015)

[13] E. J. Bergholtz, Z. Liu, M. Trescher, R. Moessner, and M. Udagawa, Topology and Interactions in a Frustrated Slab: Tuning from Weyl Semimetals to $\mathcal{C}>1$ Fractional Chern Insulators, Phys. Rev. Lett. 114, 016806 (2015).
[14] Y. Xu, F. Zhang, and C. Zhang, Structured Weyl Points in Spin-Orbit Coupled Fermionic Superfluids, Phys. Rev. Lett. 115, 265304 (2015).

[15] M. O. Goerbig, J. N. Fuchs, G. Montambaux, and F. Piechon, Tilted anisotropic Dirac cones in quinoid-type graphene and $\alpha$-(BEDT-TTF) $)_{2} \mathrm{I}_{3}$, Phys. Rev. B 78, 045415 (2008).

[16] M. Trescher, B. Sbierski, P. W. Brouwer, and E. J. Bergholtz, Quantum transport in Dirac materials: Signatures of tilted and anisotropic Dirac and Weyl cones, Phys. Rev. B 91, 115135 (2015).

[17] M. N. Ali, J. Xiong, S. Flynn, J. Tao, Q. D. Gibson, L. M. Schoop, T. Liang, N. Haldolaarachchige, M. Hirschberger, N. P. Ong, and R. J. Cava, Large, non-saturating magnetoresistance in $\mathrm{WTe}_{2}$, Nature 514, 205 (2014).

[18] G. Autès, D. Gresch, M. Troyer, A. A. Soluyanov, and O. V. Yazyev, Robust Type-II Weyl Semimetal Phase in Transition Metal Diphosphides $X \mathrm{P}_{2}(X=\mathrm{Mo}, W)$, Phys. Rev. Lett. 117, 066402 (2016).

[19] N. Kumar, Y. Sun, K. Manna, V. Suess, I. Leermakers, O. Young, T. Foerster, M. Schmidt, B. Yan, U. Zeitler, C. Felser, and C. Shekhar, Extremely high magnetoresistance and conductivity in the type-II Weyl semimetal $\mathrm{WP}_{2}$, arXiv:1703.04527.

[20] I. Belopolski, D. S. Sanchez, Y. Ishida, X. Pan, P. Yu, S.-Y. Xu, G. Chang, T.-R. Chang, H. Zheng, N. Alidoust, G. Bian, M. Neupane, S.-M. Huang, C.-C. Lee, Y. Song, H. Bu, G. Wang, S. Li, G. Eda, H.-T. Jeng, T. Kondo, H. Lin, Z. Liu, F. Song, S. Shin, and M. Z. Hasan, Discovery of a new type of topological Weyl fermion semimetal state in $\mathrm{Mo}_{x} \mathrm{~W}_{1-x} \mathrm{Te}_{2}$, Nat. Commun. 7, 13643 (2016).

[21] M. Udagawa and E. J. Bergholtz, Field-Selective Anomaly and Chiral Mode Reversal in Type-II Weyl Materials, Phys. Rev. Lett. 117, 086401 (2016).

[22] S. Tchoumakov, M. Civelli, and M. O. Goerbig, MagneticField-Induced Relativistic Properties in Type-I and Type-II Weyl Semimetals, Phys. Rev. Lett. 117, 086402 (2016).

[23] Z.-M. Yu, Y. Yao, and S. A. Yang, Predicted Unusual Magnetoresponse in Type-II Weyl Semimetals, Phys. Rev. Lett. 117, 077202 (2016).

[24] T. Meng, A. G. Grushin, K. Shtengel, and J. H. Bardarson, Theory of a 3+1D fractional chiral metal: Interacting variant of the Weyl semimetal, Phys. Rev. B 94, 155136 (2016).

[25] H. Wei, S.-P. Chao, and V. Aji, Excitonic Phases from Weyl Semimetals, Phys. Rev. Lett. 109, 196403 (2012).

[26] Z. Wang and S.-C. Zhang, Chiral anomaly, charge density waves, and axion strings from Weyl semimetals, Phys. Rev. B 87, 161107 (2013). 
[27] M. Laubach, C. Platt, R. Thomale, T. Neupert, and S. Rachel, Density wave instabilities and surface state evolution in interacting Weyl semimetals, Phys. Rev. B 94, 241102(R) (2016).

[28] B. Roy, P. Goswami, and V. Juričić, Interacting Weyl fermions: Phases, phase transitions, and global phase diagram, Phys. Rev. B 95, 201102(R) (2017).

[29] Y. Wang and P. Ye, Topological density-wave states in a particlehole symmetric Weyl metal, Phys. Rev. B 94, 075115 (2016).

[30] K.-Y. Yang, Y.-M. Lu, and Y. Ran, Quantum Hall effects in a Weyl semimetal: Possible application in pyrochlore iridates, Phys. Rev. B 84, 075129 (2011).

[31] B. Roy and J. D. Sau, Magnetic catalysis and axionic charge density wave in Weyl semimetals, Phys. Rev. B 92, 125141 (2015).

[32] X.-T. Zhang and R. Shindou, Transport properties of density wave phases in three-dimensional metals and semimetals under high magnetic field, Phys. Rev. B 95, 205108 (2017).

[33] X.-Q. Sun, S.-C. Zhang, and Z. Wang, Helical Spin Order from Topological Dirac and Weyl Semimetals, Phys. Rev. Lett. 115, 076802 (2015).

[34] See Supplemental Material at http://link.aps.org/supplemental/ 10.1103/PhysRevB.96.201101 for details of the mean field calculation and additional information on susceptibility and heat capacity.

[35] A. V. Chubukov, D. V. Efremov, and I. Eremin, Magnetism, superconductivity, and pairing symmetry in iron-based superconductors, Phys. Rev. B 78, 134512 (2008).

[36] M. O. Goerbig, Electronic properties of graphene in a strong magnetic field, Rev. Mod. Phys. 83, 1193 (2011).

[37] M. O. Goerbig, P. Lederer, and C. M. Smith, Competition between quantum-liquid and electron-solid phases in intermediate Landau levels, Phys. Rev. B 69, 115327 (2004).
[38] H. Fukuyama, P. M. Platzman, and P. W. Anderson, Twodimensional electron gas in a strong magnetic field, Phys. Rev. B 19, 5211 (1979).

[39] J. Knolle and N. R. Cooper, Quantum Oscillations without a Fermi Surface and the Anomalous de Haas-van Alphen Effect, Phys. Rev. Lett. 115, 146401 (2015).

[40] D. Yoshioka and H. Fukuyama, Electronic Phase Transition of Graphite in a Strong Magnetic Field, J. Phys. Soc. Jpn. 50, 725 (1981).

[41] B. Fauqué, D. LeBoeuf, B. Vignolle, M. Nardone, C. Proust, and K. Behnia, Two Phase Transitions Induced by a Magnetic Field in Graphite, Phys. Rev. Lett. 110, 266601 (2013).

[42] D. LeBoeuf, C. W. Rischau, G. Seyfarth, R. Küchler, M. Berben, S. Wiedmann, W. Tabis, M. Frachet, K. Behnia, and B. Fauqué, Thermodynamic signatures of the field-induced states of graphite, arXiv:1705.07056.

[43] S. Thirupathaiah, R. Jha, B. Pal, J. S. Matias, P. Kumar Das, P. K. Sivakumar, I. Vobornik, N. C. Plumb, M. Shi, R. A. Ribeiro, and D. D. Sarma, $\mathrm{MoTe}_{2}$ : An uncompensated semimetal with extremely large magnetoresistance, Phys. Rev. B 95, 241105(R) (2017).

[44] B. A. Bernevig, T. L. Hughes, S. Raghu, and D. P. Arovas, Theory of the Three-Dimensional Quantum Hall Effect in Graphite, Phys. Rev. Lett. 99, 146804 (2007).

[45] S. K. McKernan, S. T. Hannahs, U. M. Scheven, G. M Danner, and P. M. Chaikin, Competing Instabilities and the High Field Phases of (TMTSF) ${ }_{2} \mathrm{ClO}_{4}$, Phys. Rev. Lett. 75, 1630 (1995).

[46] L. Balicas, G. Kriza, and F. I. B. Williams, Sign Reversal of the Quantum Hall Number in (TMTSF) $)_{2} \mathrm{PF}_{6}$, Phys. Rev. Lett. 75, 2000 (1995). 